\title{
Bartholin's Gland Eyst Excision Using Hydrodissection and Bipolar Forceps Hemostasis
}

\author{
Original \\ Ramkrishna Purohit, Jay Gopal Sharma, Devajani Meher, Sanjay Raosaheb \\ Article \\ Rakh, Surya Malik
}

Department of Obstetrics and Gynecology, Purohit General Hospital, Shakti Nagar, Odisha, India

\begin{abstract}
Aim: Recurrence of Bartholin's cyst after various surgical treatments causes an unpleasant situation for the patient and the couple. To prevent recurrence, the present study described an operative procedure to excise the Bartholin's gland cyst or abscess using hydrodissection and bipolar forceps hemostasis.

Patients and methods: In a retrospective study in our private hospital, we studied cases that had undergone excision of Bartholin's gland cyst or abscess for benign indications by the described procedures.

Results: A total of 21 cases underwent excision of Bartholin's gland cyst or abscess by the described procedures. All were in the reproductive age ranged between 22 and 40 years. Seven of the total 21 cases had history of previous emergency incision and drainage to provide relief of severe vulval pain owing to Bartholin's abscess. All these seven cases had recurrence of Bartholin's cyst subsequently and had to undergo excision of the gland. Of the total 21 cases, 14 had an elective excision of Bartholin's cyst or abscess owing to vulvar swelling and dyspareunia and did not experience recurrence subsequently. None of the 21 cases developed postoperative hematoma, cellulitis, or abscess of the operation site requiring readmission. None of them had bad scarring or had subsequent dyspareunia following the operation. Median operation time was $17 \mathrm{~min}(13-23 \mathrm{~min})$. None of the total 21 cases that underwent excision of Bartholin's cyst had recurrence of cyst or abscess.

Conclusion: Hydrodissection with coagulation hemostasis may simplify successful excision of Bartholin's gland cyst to avoid recurrence.
\end{abstract}

Key Words: Bartholin's cyst, bartholin's cyst excision, bartholin's cyst recurrence, hydrodissection of bartholin's cyst.

Received: 22 April 2018, Accepted: 26 Janurary 2019

Corresponding Author: Ramkrishna Purohit, MD, Department of Obstetrics \& Gynecology, Purohit General Hospital, Shakti Nagar, Bargarh 768028, Odisha, India, Tel.: +91 6646232 596, Fax: +91 6646231597 , E-mail: ramkrishnapurohit@hotmail.com.

ISSN: 2537-0014

\section{INTRODUCTION}

Recurrence of the Bartholin's cyst and scarring of the vaginal wall following various conventional surgical procedures like drainage of an abscess or marsupialization etc., cause an unpleasant situation for the patient and the couple ${ }^{[1]}$. Infection of the Bartholin's gland cyst and formation of abscess cause severe vulval pain and dyspareunia. The excision of the Bartholin's gland cyst or abscess with layer closure causes scarring of vaginal wall and subsequent dyspareunia. To avoid scarring of the vaginal wall and recurrence following the surgical treatment ${ }^{[2,3]}$, we designed the later described operative procedure to excise the Bartholin's cyst or abscess with the gland and studied its feasibility.

\section{PATIENTS AND METHODS}

In a retrospective study in our private hospital from January 2016 through December 2017, we studied cases that received excision of Bartholin's gland cyst or abscess for benign indications. We excluded excision of other cysts of the vagina or vulva from the study.

We wanted to know the feasibility of the excision of the Bartholin's cyst or abscess by the following described procedure. Each patient signed informed consent before operation. Institutional ethics committee approved the study.

\section{Procedure}

Four drops of injection adrenaline (1 : 1000) were mixed with $40 \mathrm{ml}$ of normal saline ${ }^{[4]}$. The solution was infiltrated subcutaneously into the loose tissues around the cyst or abscess to facilitate development of the tissue dissection plane and to reduce intraoperative bleeding. Medial to the hymen fold, a linear incision of $2.5-3.0 \mathrm{~cm}$ was made by a surgical knife to incise the vaginal skin. The incision 
margins were held and stretched by Alli's forceps to make a split. The cleavage between the vaginal skin and the cyst wall was established by an artery forceps or a scissors using the open and close technique of dissection to expose the cyst wall. The exposed cyst wall was grasped by another Alli's forceps and pulled gently in medially downward direction. Then, the tip of the index finger was inserted into the cleavage between the vaginal margin and the cyst wall, and the tense cyst (Figure 1) was gradually dissected out of the loose subcutaneous and paravaginal fascia using the tactile feel. No sharp dissection was used. Vessels and tough cords of the tissue encountered on the way during the blunt dissection were separated from the cyst wall using scissors after bipolar forceps coagulation (45 W) (Figure 2) to cause bloodless enucleation. Medially downward traction of the cyst and blunt dissection by finger gradually eased complete scaling of the cyst with gland out of the loose paravaginal bed of tissues. The labial or vaginal skin was not excised at any stage of the operation. No mops were used to clean blood during dissection. Normal saline spray by a syringe of $20 \mathrm{ml}$ with suction aspiration was used to keep the operation site clean. In the case of an open cyst or an open abscess, normal saline lavage with suction aspiration was done to clear the contents. The tip of an artery forceps was used to grasp the cyst wall margin and pulled in the medially downward direction in a similar fashion as described before. The tip of the surgeon's left index finger was placed inside the lumen of cyst or abscess (Fig. 2) to define the outer limit of the cyst wall and then the blunt dissection procedures were carried out by right index finger similar to the aforementioned description.

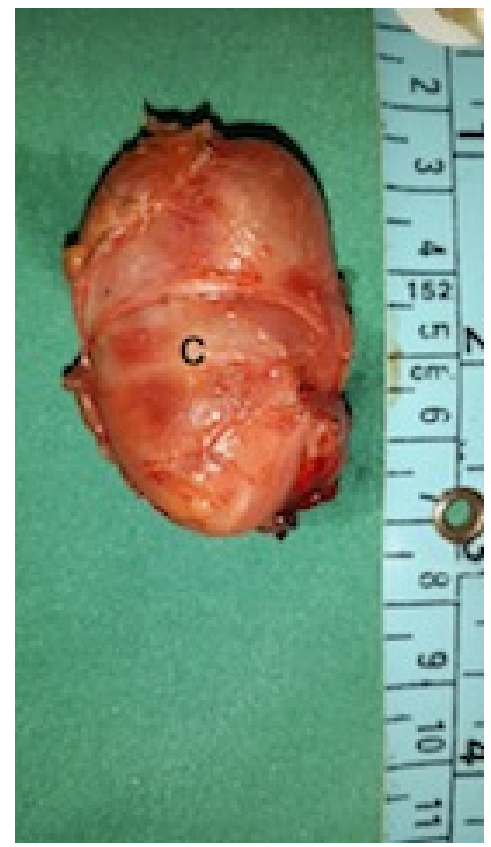

Fig. 1: Bartholin's cyst specimen following excision. C, Bartholin's cyst.

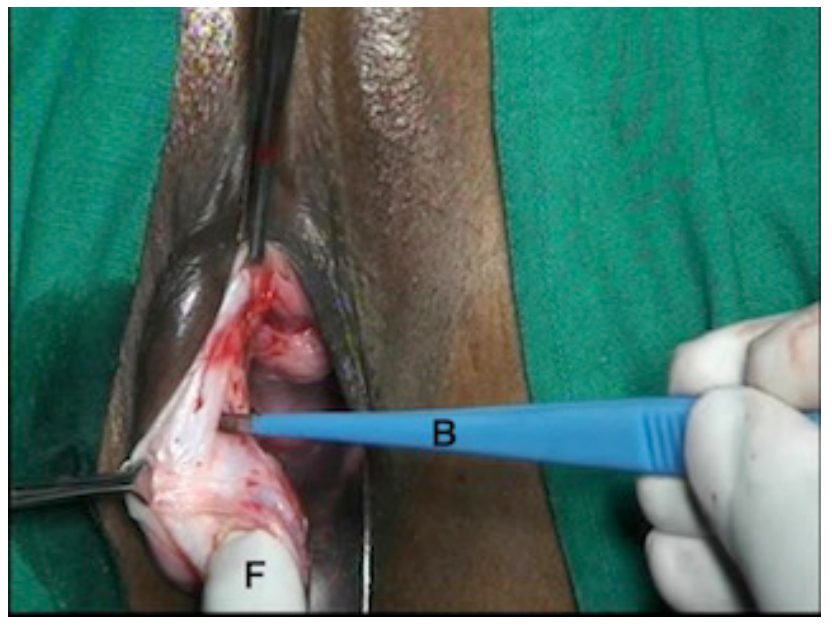

Fig. 2: Cyst wall removal procedure out of the loose paravaginal tissues. F, fingertip side the open cyst; B, bipolar forceps.

After removal of the cyst, incision margins were retracted by Alli's forceps to expose bleeder, if any, inside the wound bed and were cauterized using bipolar forceps coagulation (45 W) to achieve complete hemostasis. Suturing of wound bed in layers or closure of the wound was avoided. No gauze packing was used inside the wound to cause hemostasis, but a betadine lotion-soaked ribbon gauze pack was placed in the vagina (outside the wound) for approximately 4-6 $\mathrm{h}$ to keep both incision margins in position and to keep the wound compressed. Details of the operation procedure can be seen opening the link https://youtu. be/MQ0w6hXABDc. Postoperatively, the patient was given broad-spectrum antibiotics. She was examined per vaginally by one finger $24 \mathrm{~h}$ after the operation to find hematoma, if any and before sending her to the home. She was further advised to have sitz baths and local application of betadine ointment for 2 weeks at home. They were followed up after 6 weeks and 6 months.

\section{RESULTS}

The total number of cases was 21 . All patients were between 22 and 40 years of age and were married.

Seven of the 21 cases had history of emergency incision and drainage to provide relief of severe vulvar pain owing to Bartholin's abscess. All those seven cases developed recurrence of the cyst, and subsequently underwent excision of the gland. Fourteen of the 21 cases underwent elective excision of Bartholin's cyst or abscess owing to vulval swelling and dyspareunia. Four of those 14 cases had history of spontaneous rupture of the abscess within $24 \mathrm{~h}$ of the hospital admission. Two of the 14 cases underwent Bartholin's abscess excision during the pregnancy.

All cases underwent unilateral Bartholin's cyst gland excision by the described procedure in this study. Five cases developed postoperative pyrexia, which subsided after $48 \mathrm{~h}$ of broad-spectrum 
antibiotic administration. All cases had less pain in the postoperative period. Spontaneous apposition of the vaginal wound margins was seen $24 \mathrm{~h}$ after operation in all cases. None of the cases developed postoperative hematoma, damage to the rectum, cellulitis, or abscess of operation site requiring a readmission.

None of the cases developed severe postoperative scarring at the operation site, and subsequent dyspareunia. After 6 weeks following operation, no difference between left and right vaginal wall was observed except a light scar mark on the site of incision. Histopathological study of the excised gland did not show malignancy in any of the 21 cases. None of the cases complained of difficulty in sexual function subsequently after 6 weeks. Median operation time was $17 \mathrm{~min}$ (13-23 min). Hospital stay ranged from 1 to 2 days. None of the 21 cases reported recurrence after excision of cyst or abscess.

\section{DISCUSSION}

Infiltration of the normal saline mixed solution with injection adrenaline $(1: 1000)$ around the Bartholin's cyst caused temporary hemostasis and helped to develop soft tissue plane. The hydrodissection also assisted the progress of blunt dissection by finger using tactile feel to enucleate through the skin split the firm Bartholin's gland mass (cyst or abscess) entirely from the loose paravaginal fascia. Bipolar forceps coagulation hemostasis avoided the use of sutures to ligate bleeder if any, and thus, avoided the development of subsequent scarring of the vaginal wall and dyspareunia. In contrast to conventional surgical methods of excision, no layer closure was needed in this study as both incision margins fall on each other after removal of the space occupying subcutaneous cyst and thus avoided the development of subsequent scarring and dyspareunia.

Nonrecurrence of Bartholin's cyst after the excision indicated complete excision by the present procedure. Methods of fistulization of Bartholin's cyst, though are simple, have the disadvantages of frequent recurrence, scarring, dyspareunia, persistent drainage, and hemorrhage ${ }^{[1,5]}$. Similar to findings by another study ${ }^{[6]}$, the present one-time initial surgical excision treatment of the Bartholin's gland in either cyst or abscess stage could be done. It could avoid botherations of recurrence reported by other studies ${ }^{[2,3,7]}$. Excision using bipolar hemostasis avoided layer closure and formation of subsequent scarring of the vaginal wall and dyspareunia.

\section{CONCLUSION}

Hydrodissection with bipolar coagulation hemostasis may simplify successful excision of Bartholin's gland cyst to avoid the recurrence.

\section{CONFLICTS OF INTEREST}

There are no conflicts of interest.

\section{REFERENCES}

1. ReifP,Ulrich D, Bjelic-Radisic V. Management of Bartholin's cyst and abscess using the Word catheter: implementation, recurrence rates and costs. Eur J Obstet Gynecol Reprod Biol 2015; 190:81-84.

2. Ozdegirmenci O, Kayikcioglu F, Haberal A. Prospective randomized study of marsupialization versus silver nitrate application in the management of bartholin gland cysts and abscesses. J Minim Invasive Gynecol 2009; 16:149-152.

3. Wechter ME, Wu JM, Marzano D, Haefner H. Management of Bartholin duct cysts and abscesses: a systematic review.Obstet Gynecol Surv 2009; 64:395-404.

4. Purohit RK. Purohit technique of vaginal hysterectomy.a new approach. BJOG 2003; 110:1115-1119.

5. Gennis P, Li SF, Provataris J. Jacobi ring catheter treatment of Bartholin's abscesses. Am J Emerg Med 2005; 23:414-415.

6. Kallam AK, Kanumury V, Bhimavarapu N. A report of two cases of 'giant bartholin gland cysts' successfully treated by excision with review of literature. J Clin Diagn Res 2017; 11:pd11-pd13.

7. Lee MY, Dalpiaz A, Schwamb R. Clinical pathology of Bartholin's glands: a review of the literature. Curr Urol 2015; 8:22-25. 